\title{
Paediatric HRCT of the chest - help for the general radiologist
}

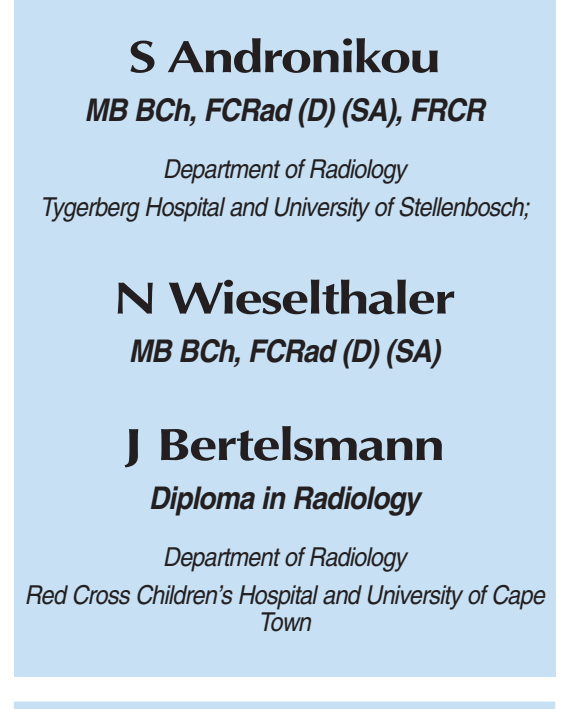

\section{Introduction}

A higher proportion of paediatric interstitial lung diseases can be diagnosed with high-resolution computed tomogra- phy (HRCT) scan than plain radiography, with the diagnosis being made with more confidence and higher accuracy. ${ }^{1}$ HRCT is probably easier to interpret in the child than in the adult. There is less pressure to reach a definitive diagnosis from the HRCT alone, because many entities resulting in diffuse lung disease are rare in childhood and do not require consideration. In addition, endstage fibrosis, which has nonspecific appearances, is rare. Lastly, infection is the most likely cause of most positive studies.

\section{When to perform a HRCT (indications are similar to those in adults $)^{2,3}$}

(i) When the patient is symptomatic but the chest X-ray (CXR) is normal; (ii) to confirm an interstitial pattern seen on CXR; (iii) to determine the severity of disease; (iv) to look for bronchiectasis; $(v)$ to look for predisposing factors; ( $v i$ ) to identify the main abnormality and possibly come close to a diagnosis; (vii) to look for sequelae of infection; and (viii) to show a site for biopsy.

\section{How to approach $\mathrm{HRCT}^{4}$}

Look for the following:

Airway disease: nodules, mosaic pattern, bronchiectasis, bronchial wall thickening, and air trapping.

Airspace disease: nodules, ground glass, mosaic pattern, and consolidation.

Interstitial disease: nodules, ground glass, mosaic pattern, septal thickening, parenchymal bands, air-filled cysts, honeycombing, and architectural distortion.

\section{Features on HRCT}

\section{Nodules}

Nodules are focal oval densities of a variety of sizes that can be 'well defined' or 'ill defined' and are interpreted according to their predominant distribution and size (Table I).

A good example of 'fluffy' or ill-defined

Table I. Causes of pulmonary nodules in children ${ }^{3,4}$

\begin{tabular}{|c|c|c|c|}
\hline Small ill-defined CLOs & Small soft-tissue nodules & Larger masses & Halo sign \\
\hline Bronchogenic $\mathrm{TB}^{\star}$ & Miliary TB & $\mathrm{TB}$ & TB \\
\hline Aspiration* & Fungi & Fungi & Pulmonary aspergillosis \\
\hline Bronchiectasis ${ }^{*}$ & Metastases & Metastases & Lymphoproliferative \\
\hline Asthma* & Langerhans cell histiocytosis & Septic emboli & Wegener's granulomatosis \\
\hline Cystic fibrosis* & Pulmonary haemosiderosis & Lymphoma & Pulmonary haemorrhage \\
\hline \multicolumn{2}{|l|}{ Infective bronchiolitis ${ }^{\star}$} & AVM & Osteosarcoma metastases \\
\hline \multicolumn{2}{|l|}{$\operatorname{LIP}^{\star}$} & $\mathrm{LCH}$ & \\
\hline \multicolumn{2}{|l|}{$\mathrm{LCH}$} & Papillomatosis & \\
\hline \multicolumn{2}{|c|}{ Hypersensitivity pneumonitis } & Vasculitis & \\
\hline \multicolumn{2}{|c|}{ Pulmonary capillary haemoangiomatosis } & $\mathrm{BOOP} / \mathrm{COP}$ & \\
\hline \multicolumn{2}{|c|}{ Pulmonary haemosiderosis } & Pulmonary arterial aneurysm & \\
\hline \multicolumn{2}{|c|}{ Follicular bronchiolitis } & Bleomycin & \\
\hline \multicolumn{2}{|l|}{ BO } & Lipoid granuloma (TPN) & \\
\hline \multicolumn{4}{|c|}{ Congenital lymphangiectasia } \\
\hline \multicolumn{4}{|c|}{ Immotile cilia } \\
\hline
\end{tabular}


nodules is in pulmonary oedema where they tend to coalesce and form areas of consolidation. True interstitial nodules are 'well-defined' and are well represented by military tuberculosis (TB) (Fig. 1a and b). Even though there are innumerable nod-

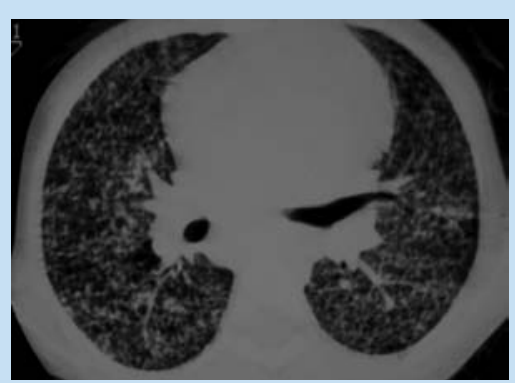

Fig. 1a. Miliary TB.

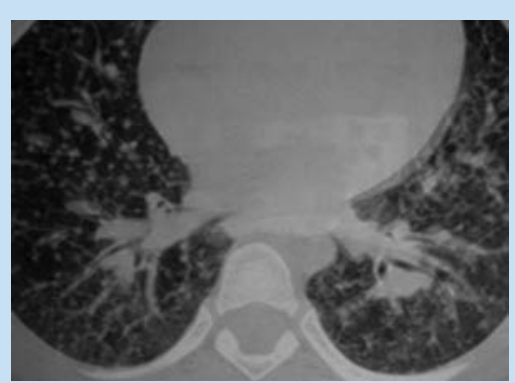

Fig. 1b. Miliary TB.

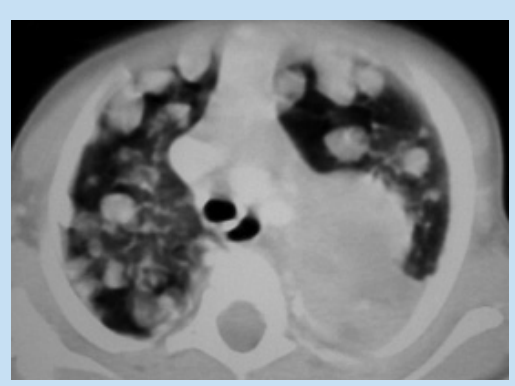

Fig. 2a. Metastases.

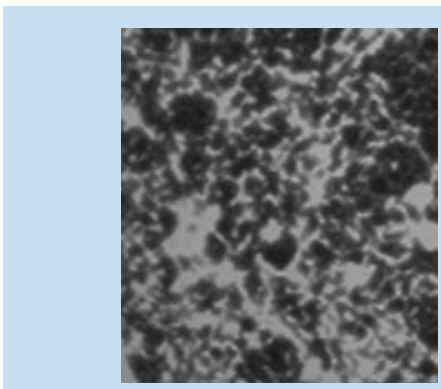

Fig. 2b. Centrilobar opacity (CLO). ules, they remain discreet and 'can be picked off the page individually with tweezers'. Nodules of varying sizes, which are randomly distributed, especially in the periphery and usually affect both lungs, are characteristic of metastatic disease (Fig. 2a). A special type of nodule is the centrilobular opacity (CLO). These are about $5 \mathrm{~mm}$ in size and are seen within the secondary lobule as nodules, branching ' $\mathrm{Y}$ ' structures or 'trees in bud' (Fig. 2b). They represent material within the central bronchiole of the secondary lobule. . $^{3,4}$

\section{Ground glass opacification (GG) and consolidation}

GG is an increased density of the lung where the vascular and bronchial markings are still visible. There may be air bronchograms. Causes include filling of air spaces, thickening of the interstitium, partial collapse of alveoli, expiration in a normal individual or increased capillary volume. GG is seen regularly with infections and is an important but nonspecific HRCT feature of the chronic diffuse interstitial pneumonitides (Table II) (Fig. 3). GG surrounding a nodule results in the 'halo sign', often seen in fungal disease (Fig. $4 \mathrm{a}$ and b). ${ }^{4}$

\section{Table II. Ground glass / consolidation ${ }^{3,4}$}

Oedema $^{*}$

Pneumonia*

Respiratory distress syndrome*

Leukaemia*

Contusion*

Pulmonary haemorrhage $e^{\star}$

Alveolar proteinosis

Drug toxicity

Extrinsic allergic alveolitis

Transplant rejection

Sarcoid

Collagen vascular disease

Post lavage

Idiopathic interstitial pneumonitides (NSIP)

Idiopathic pulmonary fibrosis

${ }^{*}$ Common causes.

$\mathrm{NSIP}=$ nonspecific interstitial pneumonitis

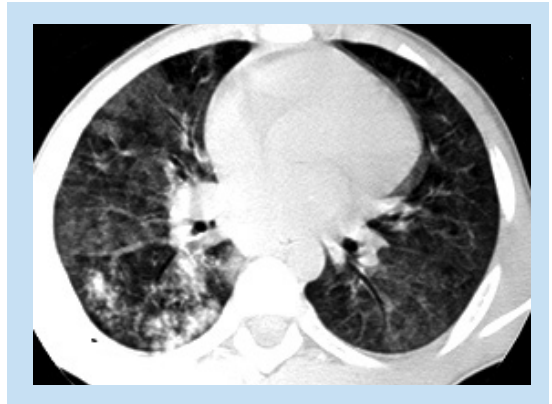

Fig. 3. Ground glass (GG).

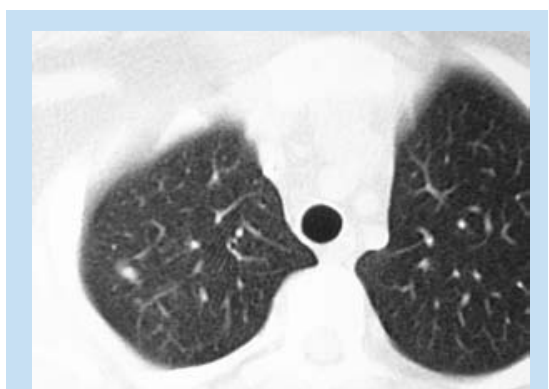

Fig. 4a. Halo sign

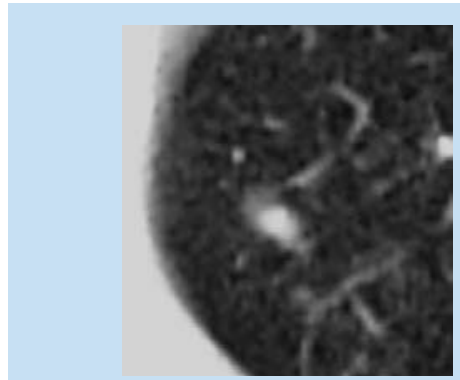

Fig. 4b. Zoomed 'halo sign'.

Consolidation is caused by filling of the alveoli with fluid or cells or both and differs from GG in that the increased density obscures vascular markings and is usually accompanied by air bronchograms (Figs 5 and 6).

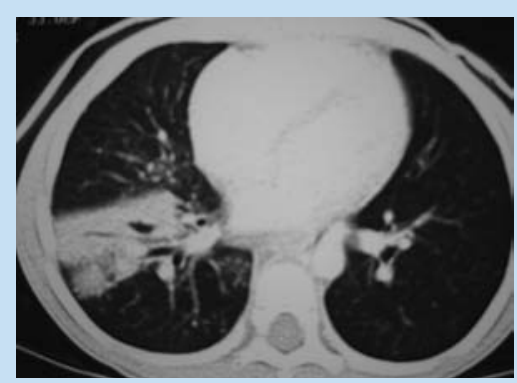

Fig. 5. Lobar airspace opacification. 


\section{PICTORIAL ESSAY}

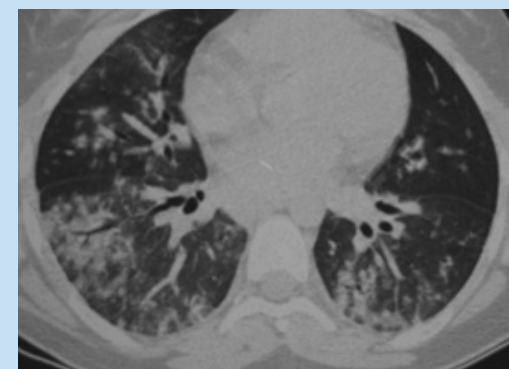

Fig. 6. Patchy airspace opacification.

Tip: Focal GG is usually due to air space disease or a vasculitis and diffuse GG is usually representative of an interstitial process. $^{2-4}$

Tip: GG can cause a 'mosaic pattern' on HRCT and requires an expiratory scan to distinguish it from air-trapping. ${ }^{2-}$

\section{Mosaic pattern and air-trap- ping (Table III)}

'Mosaic pattern' is a geographic pattern of varying lung density. It reflects either focal areas of air-trapping (a sign of small airways disease) (Fig. 7a) but is also associated with focal areas of GG (Fig. 7b). The two entities can be differentiated by an expiratory HRCT in which the low-density areas become more exaggerated and vessel calibre decreases if the mosaic pattern is due to air-trapping. Mosaic perfusion is seen with bronchiolitis obliterans and bronchiectasis.

\begin{tabular}{|l|}
\hline \multicolumn{1}{|c|}{ Table III. Air-trapping } \\
\hline Bronchiolitis obliterans \\
Cystic fibrosis \\
Bronchiectasis \\
Asthma \\
Viral \\
Left to right \\
Follicular bronchial hyperplasia \\
\hline
\end{tabular}

Generalised air-trapping may be due to partial or complete airway obstruction or local abnormality in pulmonary compliance. Measuring the Hounsfield density of the lung can assess this. Normal lung measures -600 to $-750 \mathrm{HU}$ while air-trapping results in density measurements of -900 HU. It can also be assessed with an expira- tory view, which in small children is obtained by a decubitus view of the 'sidedown' hemithorax (Fig. 8).,3,

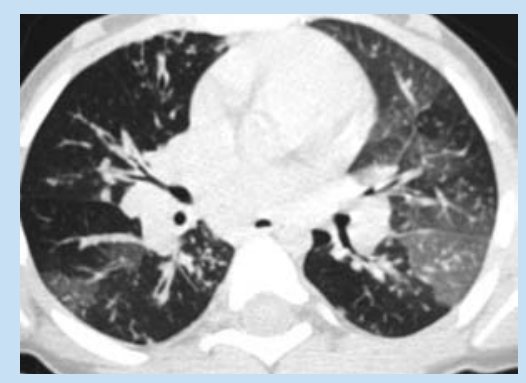

Fig. 7a. Mosaic pattern due to air-trapping.

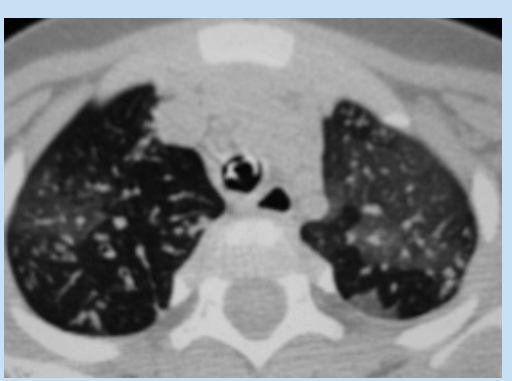

Fig. 7b. Mosaic pattern due to focal areas GG

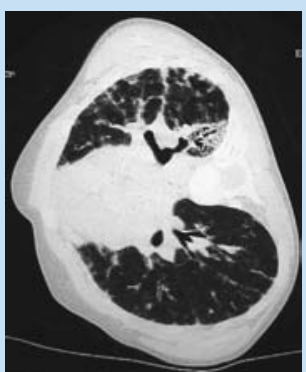

Fig. 8. Air-trapping in 'side down' lung.

\section{Septal lines}

This is abnormal widening of the interlobular septa and can either be seen as linear densities perpendicular to and touching the pleura (equivalent to Kerley B lines) or as a polygonal pattern outlining the secondary lobule more centrally (Fig. 9a and b). ${ }^{4}$ These are often seen with lymphatic involvement in diseases such as lymphangiomatosis and lymphangitic spread of malignancy. ${ }^{5}$ The polygonal pattern of linear densities on a background of GG results in the 'crazy paving' pattern best known to occur in alveolar proteinosis. ${ }^{4}$ Lines may be smooth or irregular helping to differentiate some of the causes of this feature (Table IV).

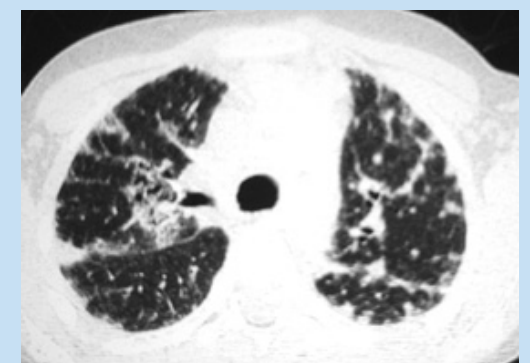

Fig. 9a. Septal lines.

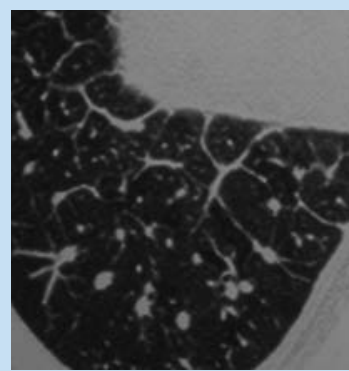

Fig. 9b. Septal lines.

\section{Parenchymal bands / archi- tectural distortion / honey- combing}

These findings all represent some form of chronic involvement and scarring / fibrosis of the lung. Parenchymal bands are longer than septal lines measuring between 2 and $5 \mathrm{~cm}$ (Fig. 10) and are often associated with a pleural 'tag. They can occur with fibrosis and scarring or atelectasis associated with fibrosis and are most often seen in children with bronchopulmonary dysplasia (chronic lung disease). Architectural distortion indicates displaced bronchi, vessels and or fissures and is usually associated with decreased vascularity and bronchial abnormalities (Fig. 11a). Honeycombing is rare in children and represents destroyed lung that becomes cystic and fibrotic (Fig. 11b). HRCT shows air-filled cysts that tend to occur in a subpleural distribution over several layers and are unaffected by expiration. This is seen in children with chronic diffuse idiopathic pneumonitides and connective tissue diseases. 


\section{PICTORIAL ESSAY}

\begin{tabular}{|lll|}
\hline \multicolumn{3}{c|}{ Table IV. Septal lines ${ }^{3,4}$} \\
\hline Smooth & Nodular / irregular & Crazy paving \\
\hline Pulmonary oedema $^{*}$ & Lymphoma & Alveolar proteinosis \\
Infection & Sarcoma / neuroblastoma & Lipoid pneumonia \\
BPD $^{*}$ & Sarcoid & ARDS \\
Pulmonary alveolar proteinosis & Fibrosis & AIP \\
Neoplasms & & Drug-induced pneu- \\
& & monia \\
Pulmonary lymphangiectasia & & \\
Lymphangiomatosis & \\
Pulmonary capillary haemangiomatosis & \\
Gaucher / Nieman Piek & \\
Sarcoid & \\
Pulmonary alveolar microlithiasis & \\
Pulmonary haemosiderosis & \\
Tuberous sclerosis & \\
Collagen vascular diseases & \\
Fibrosis & \\
BPD = bronchopulmonary dysplasia; ARDS = acute respiratory distress syndrome; AIP = acute interstitial pneumonitis.
\end{tabular}

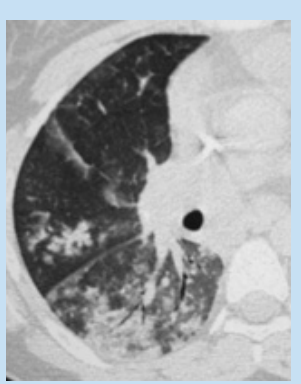

Fig. 10. Parenchymal bands.

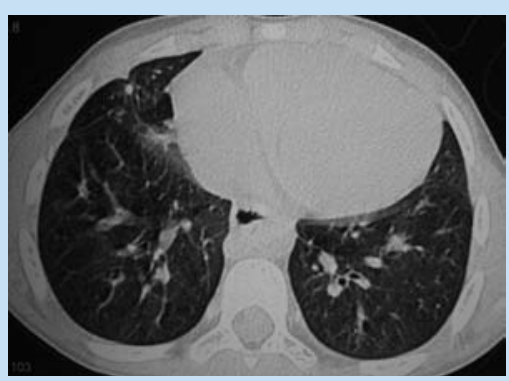

Fig. 11a. Architectural distortion

\section{Bronchiectasis}

Bronchiectasis can either be a diagnosis or a feature of another disease. It indicates irreversible dilation of a bronchus and the signature feature is the 'signet ring sign'

\begin{tabular}{|l}
\hline Table V. Causes of bronchiectasis ${ }^{3,6,8}$ \\
\hline Infection \\
TB \\
AIDS \\
Adenovirus \\
Immunodeficiency \\
Aspiration \\
Recurrent infection \\
Sequelae of infection \\
Obstruction \\
Foreign body \\
Neoplasm \\
Lymphadenopathy \\
Cystic fibrosis (most common cause in \\
children) \\
Allergic bronchopulmonary aspergillo- \\
sis \\
Ciliary dyskinesia \\
Fibrosis \\
Williams-Campbell syndrome
\end{tabular}

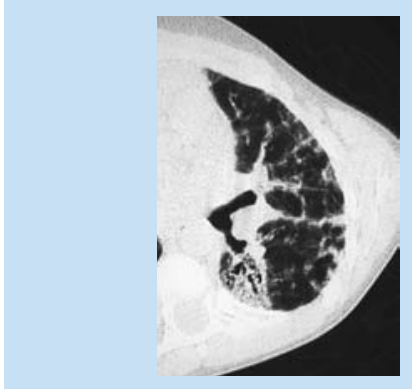

Fig. 11b. Honeycombing.

(Fig. $12 \mathrm{a}$ and b). This represents a thickwalled bronchus, which is larger than the adjacent pulmonary artery. ${ }^{4,6}$ Other indicators of bronchiectasis include non-tapering of a bronchus, bronchial wall thickening (Fig. 12b) (more than a fine 'pencil-thin' line) and visualisation of a bronchus within $1 \mathrm{~cm}$ of the periphery (including the fissures). ${ }^{7}$ Associated with bronchiectasis are atelectasis, mosaic pattern and CLOs. ${ }^{8}$ Airfluid levels may also be present within dilated bronchi. The causes of bronchiectasis are many but are most commonly associated with previous, current or recurrent infection, cystic fibrosis and aspiration (Table V).

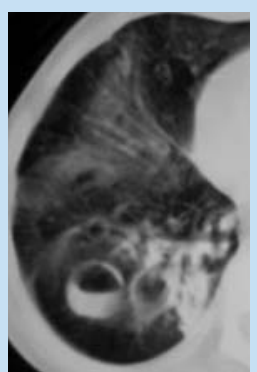

Fig.12a. Bronchiectasis.

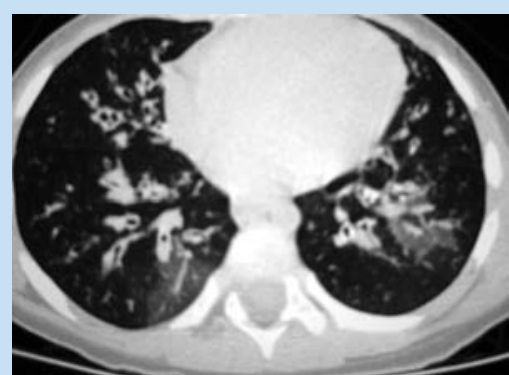

Fig.12b. Bronchial wall thickening and 'signet ring sign'. 


\section{Air-filled cysts}

These are usually thin-walled and contain air. They may be oval with smooth walls as in tuberous sclerosis or have irregular margins as in Langerhans cell histiocytosis (Fig. 13). The causes of air-filled cysts in children are listed in Table VI.

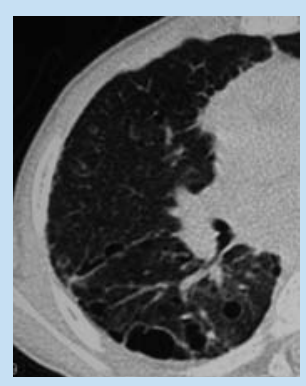

Fig. 13. Air-filled cysts in Lagerhans cell histiocytosis.

\begin{tabular}{|l|}
\hline \multicolumn{1}{|c|}{ Table VI. Air-filled cysts $^{4}$} \\
\hline Pneumatoceles ${ }^{*}$ (infective, trauma, tox- \\
ins) \\
Pneumonia* $^{*}$ \\
Septic emboli* \\
Congenital $^{*}$ \\
Tuberous sclerosis $^{*}$ \\
LCH $^{*}$ \\
Papillomatosis \\
Pseudocysts (barotrauma) \\
Wegener's granulomatosis \\
Ehlers-Danlos syndrome \\
Marfan's syndrome \\
Williams-Campbell syndrome \\
*Common causes. \\
LCH = Langerhans cell histiocytosis.
\end{tabular}

\section{Specific diseases seen in children on HRCT}

The range of diseases in children differs from adults (Table VII) ${ }^{1,5}$ and the reported accuracy for a confident first choice diagnosis on HRCT varies from $56 \%$ to $61 \%{ }^{2,5}$ The diseases diagnosed with the highest degree of confidence in children include alveolar proteinosis, pulmonary lymphangiectasia and idiopathic pulmonary haemosiderosis. ${ }^{1,2}$ We will describe the
HRCT appearances of the acute infective causes of diffuse lung diseases and diseases affecting the airways that involve the interstitium, because these occur most commonly in our practice. We will also review the appearances of those conditions characterised by nodules and septa that are seen less often in paediatric practice.

\section{Infections}

Infection - tuberculosis

Miliary nodules are common but not always present, measure $2-3 \mathrm{~mm}$ and remain discreet (Figs $1 \mathrm{a}$ and b, and 14). Bronchogenic spread of TB may result in larger nodules that may confluence. Both GG and consolidation are regular features of primary TB. CLOs, bronchiectasis and air trapping also occur. In addition, the important characteristic association is hilar and mediastinal lymphadenopathy which are the fingerprint of primary TB. ${ }^{9}$

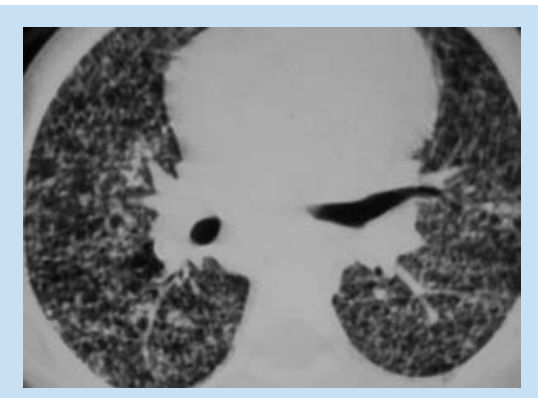

Fig. 14. Miliary TB.

Infection - AIDS: LIP (lymphoid interstitial pneumonitis)

LIP is an AIDS-defining feature in children $<13$ years of age. Between $30 \%$ and $40 \%$ of children with AIDS have LIP. HRCT features involve septal lines and nodules (CLOs and subpleural) (Fig. 15). GG, cysts and bronchiectasis are common associations. Hilar lymphadenopathy and thymic cysts may or may not be present. Effusions are not a feature.

\section{Infection - AIDS: Pneumocystis carinii}

This begins as a patchy airspace or GG appearance and progresses to a more homogeneous GG or airspace process that involves both lungs. In addition there are septa and cysts. Effusions are not a feature and there is a notable absence of nodules and lymphadenopathy. ${ }^{3}$

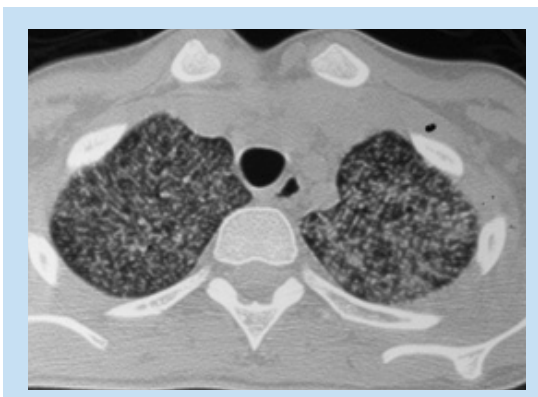

Fig.15. Lymphoid interstitial pneuomonitis.

\section{Infection - invasive aspergillosis}

This occurs in immunocompromised patients. The most characteristic features are nodules with a 'halo' sign that may cavitate (50\%) (Figs 4 and 16) and result in the 'air crescent' sign as air collects between normal and infected lung. More widespread GG or airspace disease is also noted. ${ }^{3}$ Bone marrow transplant children may require a HRCT to exclude this disease process.

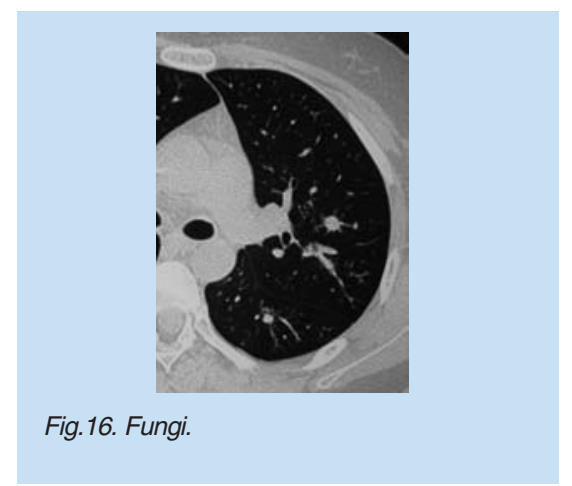

\section{Airways (Table VIII)}

\section{Airways - cystic fibrosis}

HRCT is performed to document the presence and extent of disease. The hallmark is widespread bronchiectasis involving the upper zones (Fig. 12b). Associated features such as peribronchial thickening, CLOs, larger mucoid impactions and mosaic pattern are common. ${ }^{3,6,8}$

\section{Airways - immotile cilia syndrome}

Half of these patients have Kartagener's 
Table VII. Diffuse lung diseases in children ${ }^{2,3,8}$

\begin{tabular}{llll}
\hline Common & Seen in practice & Rare & Infant-specific \\
\hline Infection & LCH & Extrinsic allergic alveolitis & Persistent tachypnoea of infancy \\
TB & Pulmonary lymphangiectasia & Sarcoid & BPD \\
Pneumocystis & Pulmonary haemorrhage & Intersitial pneumonitides & Cellular interstitial pneumonia \\
Viral & Alveolar proteinosis & Collagen vascular diseases & Infantile pulmonary haemosiderosis \\
Mycoplasma & Collagen vascular disease & Vasculitides & Chronic pneumonitis of infancy \\
Aspergillosis & Neurocutaneous syndromes & Lymphangiomatosis & Surfactant protein B deficiency \\
Chronic/recurrent & - & Alveolar microlithiasis & Familial DIP \\
LIP (AIDS-related $)$ & & Gauchers / Nieman Piek & Idiopathic pulmonary fibrosis infancy \\
BPD & & Pulmonary infiltrates with & \\
Aspiration & eosinophilia & \\
LIP = lymphoid interstitial pneumonitis; BPD $=$ bronchopulmonary dysplasia DIP = desquamative interstitial pneumonitis LCH=Langerhans cell histiocytosis.
\end{tabular}

\begin{tabular}{|c|}
\hline $\begin{array}{l}\text { Table VIII. Airway diseases in } \\
\text { children }^{1-4,8}\end{array}$ \\
\hline Infection \\
\hline Sequelae of infection \\
\hline Bronchiectasis \\
\hline Cystic fibrosis \\
\hline $\begin{array}{l}\text { Allergic bronchopulmonary aspergillo- } \\
\text { sis and asthma }\end{array}$ \\
\hline Aspiration \\
\hline Foreign body \\
\hline Swyer James / Macleod's syndrome \\
\hline $\begin{array}{l}\text { Bronchiolitis obliterans / BOOP/ follic- } \\
\text { ular bonchiolitis }\end{array}$ \\
\hline Immotile cilia \\
\hline BOOP = bronchiolitis obliterans organising pneumonia. \\
\hline
\end{tabular}

syndrome (situs inversus, sinusistis and bronchiectasis). Features cannot be differentiated from cystic fibrosis but there is a predilection for the right middle lobe. ${ }^{8}$

\section{Airways - allergic bronchopulmonary aspergillosis}

This is a hypersensitivity reaction to endobronchial growth of Aspergillus fumigatus and results in asthma. The main feature is central bronchiectasis, which in a patient with asthma, clinches the diagnosis. ${ }^{8}$
Airways - Swyer-James / Macleod's syndrome

This is a post-infectious bronchiolitis and signs include a unilateral lucent lung with air-trapping. In reality on HRCT 50\% of these patients have bilateral disease with the most affected lung showing low density, mosaic pattern and bronchiectasis, and the less affected lung showing patchy air-trapping. ${ }^{3.8}$

Airways - bronchiolitis obliterans / constrictive bronchiolitis

As expected for diseases of the airways the predominant HRCT findings are bronchial wall thickening and bronchiectasis with the associated mucoid impaction and CLOs and resultant mosaic pattern of air-trapping. ${ }^{3,4,8}$ The causes of bronchiolitis obliterans are listed below (Table IX). Bronchocentric granulomatosis has the above features but also shows septal thickening. ${ }^{3}$

Airways - BOOP (bronchiolitis obliterans organising pneumonia) and COP (cryptogenic organising pneumonia)

This is rare in children but is characterised by patchy consolidation or a GG pattern (Fig. 17a and b). Bronchial wall thickening and bronchiectasis are also features (Fig. 17c). Nodules and septa may be seen. Small effusions may also be present. ${ }^{6,8}$ The causes of BOOP are listed in Table IX.

\begin{tabular}{l} 
Table IX. Causes of bronchiolitis oblit- \\
erans and bronchiolitis obliterans with \\
organising pneumonia ${ }^{3,8}$ \\
\hline Idiopathic \\
Infection \\
Viral (adenovirus, influenza, measles) \\
Bacteria \\
Mycoplasma \\
Transplant (heart/lung $50 \%$ and bone \\
marrow $10 \%$ ) \\
Collagen vascular diseases \\
Toxic fumes \\
Stevens-Johnson syndrome \\
Post chemotherapy
\end{tabular}

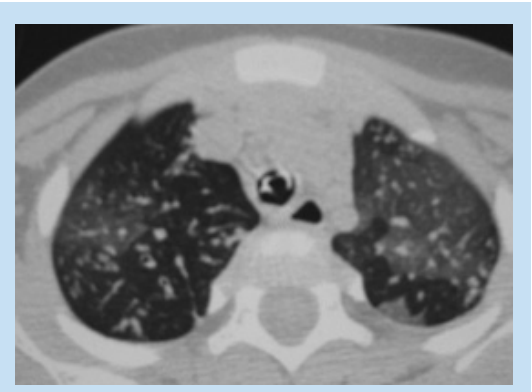

Fig.17a. Bronchiolitis obliterans. 


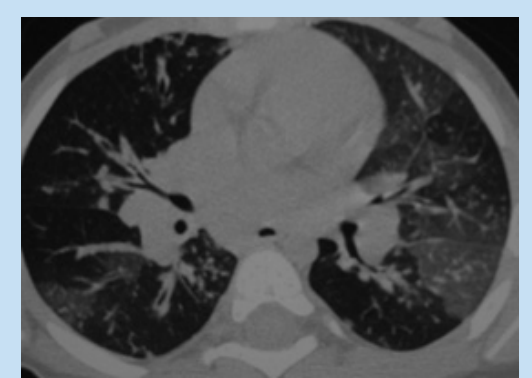

Fig. 17b. Bronchiolitis obliterans.

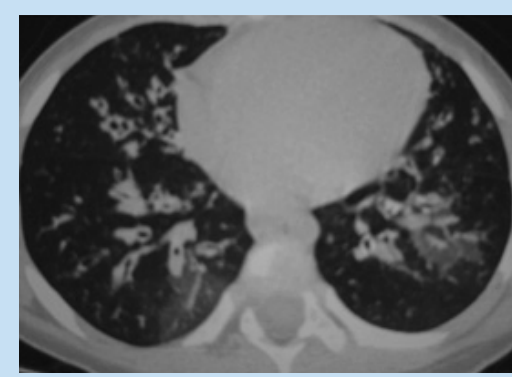

Fig. 17c. Brochiolitis obliterans.

\section{Interstitial disease: nodules and lines}

Nodules and lines - bronchopulmonary dysplasia / chronic lung disease

This occurs as a result of the treatment of premature lungs. Early appearances are characterised by septa, parenchymal bands and cystic spaces forming a 'cobblestone' pattern. Later in life a mosaic pattern and architectural distortion are present and even later there is associated bronchiolitis obliterans and traction bronchiectasis.

Nodules and lines - Langerhans cell histiocytosis

The predominant features are nodules that cavitate and become cysts. These then become thin-walled and fuse. This results in an irregular cystic appearance and is often complicated by a pneumothorax (Fig. 18). Early on in the disease, the costophrenic angles are spared. ${ }^{2,3,5}$ The other causes of air-filled cysts in the lungs are listed below (Table VI).

\section{Nodules and lines - alveolar proteinosis}

This is characterised by septa on a background of GG resulting in an easily recognisable geographic 'crazy paving' sign (Fig. 19a and b).,3

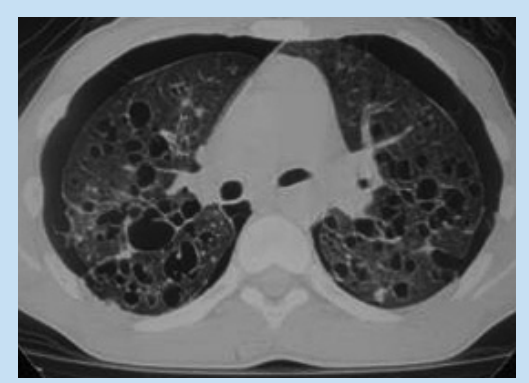

Fig. 18. Langerhans cell histiocytosis with bilateral pneumothoraces.
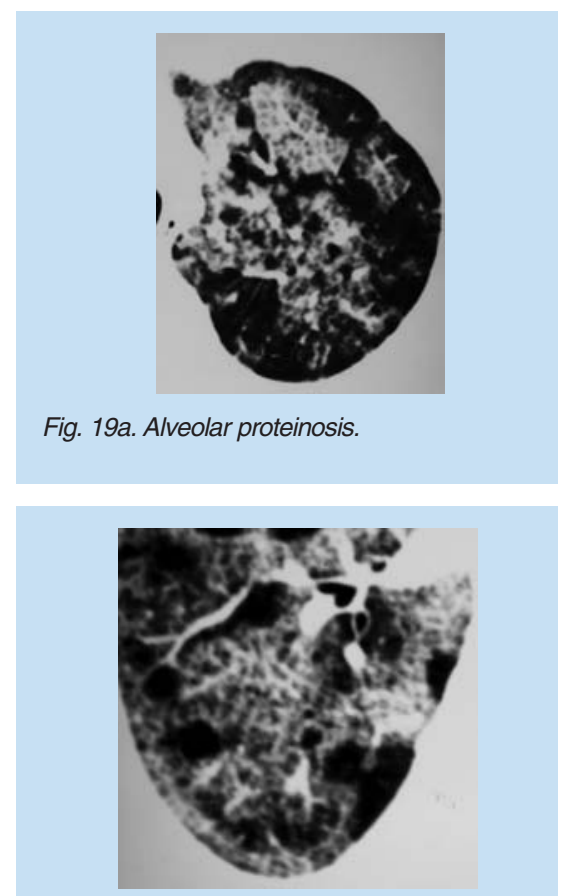

Fig. 19b. Alveolar proteinosis 'crazy paving'.

Nodules and lines - lymphangiectasia (Noonan's) / lymphangiomatosis

Here HRCT shows septal thickening and GG often associated with effusions (Fig. 20a and b). 2,3,5

Nodules and lines - lymphangitis carcinomatosa

This is a rare finding in children but can occur with lymphoma, neuroblastoma, sarcomas and thyroid malignancy. The main feature is septa accompanied by fissural and bronchovascular bundle thickening (Fig. 21a and b). It differs from sarcoid where the fissures are beaded with nodules. $^{3}$

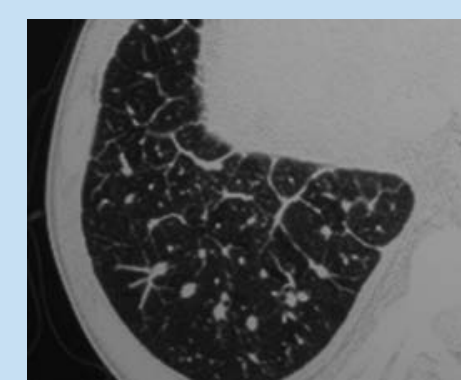

Fig. 20a. Noonan's.

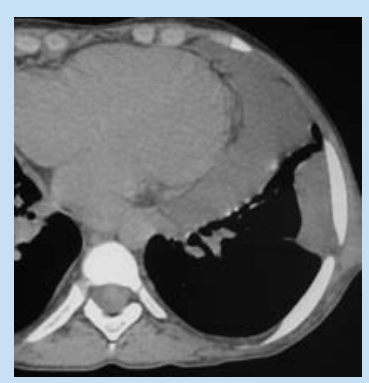

Fig. 20b. Noonan's

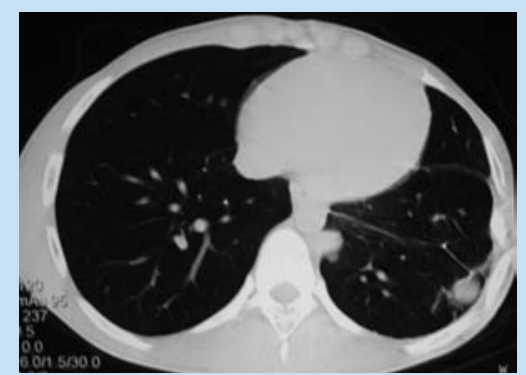

Fig. 21a. Lymphangitis carcinomatosa.

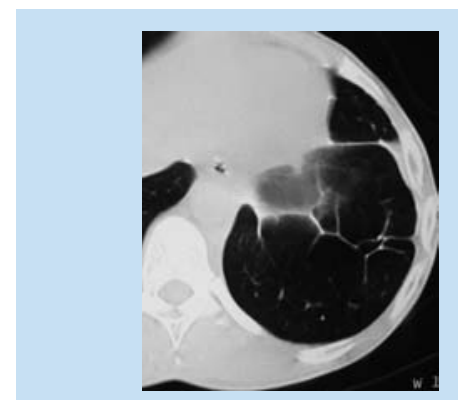

Fig. 21b. Lymphangitis carcinomatosa. 
Nodules and lines - extrinsic allergic alveolitis / hypersensitivity pneumonitis

Patchy or diffuse GG, mosaic pattern and CLOs are the prominent HRCT features. ${ }^{2,3,5}$

Nodules and lines - pulmonary haemorrhage / idiopathic pulmonary haemosiderosis

Acute haemorrhage appears either as GG or denser consolidation and is usually bilateral on HRCT (Fig. 22 a - c). ${ }^{3,10}$ More chronic haemorrhage results in nodules (CLOs and other) and septa. ${ }^{2,10}$ The causes of pulmonary haemorrhage in children are listed below (Table X).

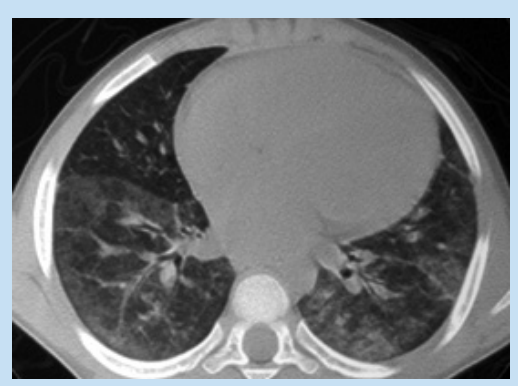

Fig. 22a. Idiopathic pulmonary haemosiderosis.

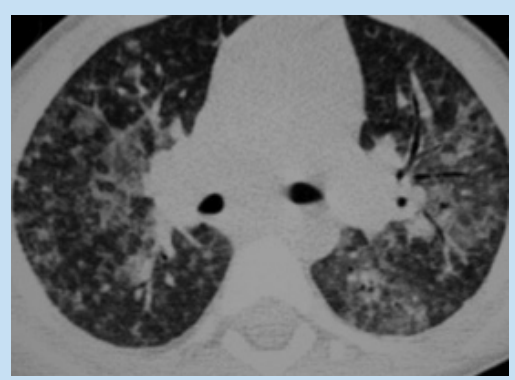

Fig. 22b. Idiopathic pulmonary haemosiderosis.

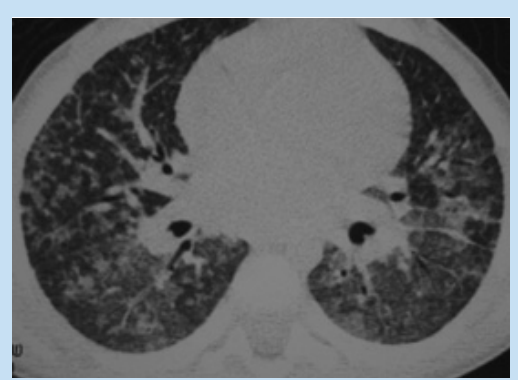

Fig. 22c. Idiopathic pulmonary haemosiderosis.

\begin{tabular}{l}
$\begin{array}{l}\text { Table X. Causes of diffuse pulmonary } \\
\text { haemorrhage in children }\end{array}$ \\
\hline Idiopathic pulmonary haemorrhage \\
Heiner's syndrome \\
Stacybotrys atra \\
Diffuse alveolar haemorrhage with \\
glomerulonephritis \\
Wegener's granulomatosis \\
Systemic necrotic vasculitis \\
Goodpasture's syndrome \\
Systemic lupus erythematosis \\
Henoch-Schonlein purpura \\
Idiopathic glomerulonephritis and \\
alveolar haemorrhage \\
Cardiovascular causes \\
Pulmonary venous hypertension \\
Mitral stenosis \\
Eisenmenger's \\
Congenital pulmonary vein stenosis \\
Congenital pulmonary artery stenosis \\
Pulmonary capillary haemangiomas
\end{tabular}

\section{Nodules and lines - sarcoid}

The nodules in sarcoid are described as 'beeding' the fissures, bronchovascular bundles and pleura (Fig. 23). Larger dense nodules may represent confluent areas of GG or consolidation, which may cavitate. Lymphadenopathy is a major feature. ${ }^{3}$

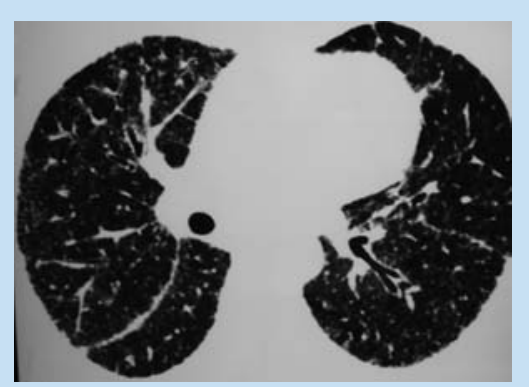

Fig. 23. Sarcoid with 'beeding'.

Nodules and lines - systemic sclerosis (vasculitis)

Even though vasculitides are rare in children, systemic sclerosis may result in lung changes on HRCT in childhood. The
HRCT features are a GG pattern with subpleural nodules that may progress to supleural cysts and honeycombing. Septa are not a feature. ${ }^{2,3}$

\section{Drug-related toxicity and injury}

Chemotherapy and other insults to the lung may result in airspace and interstitial disease, which may range from GG to septal lines and nodules to endstage fibrosis (Fig. 24).

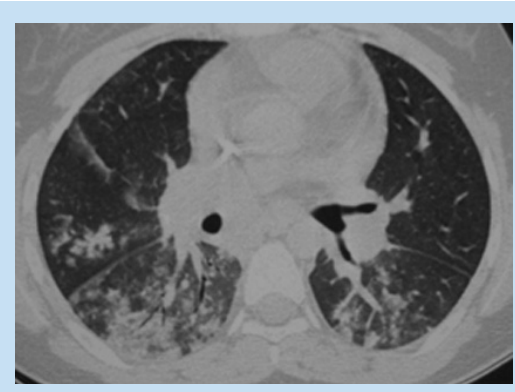

Fig. 24. Drug toxicity

\section{Endobronchial papillomatosis}

Endobronchial papillomas cause obstruction with bronchial wall thickening and dilation (Fig. 25).

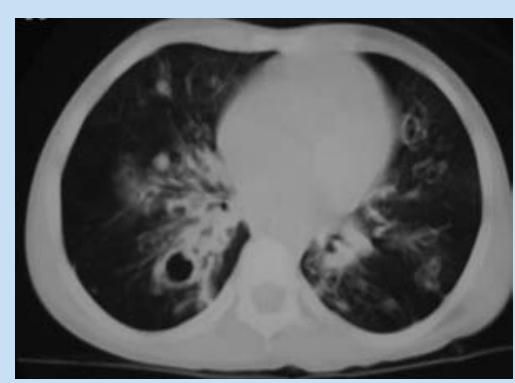

Fig. 25. Papillomatosis

Idiopathic / chronic interstitial pneumonitides and idiopathic pulmonary fibrosis

These diseases are rare in children and are distinguished only after biopsy. HRCT is less accurate in the diagnosis. ${ }^{1}$ They are predominated on HRCT by GG (Fig. 26) that progresses to pulmonary fibrosis (IPF). In children the diagnoses of DIP (desquamative interstitial pneumonitits) and UIP (usual interstitial pneumonitis) should not be listed in the differential diag- 


\section{PICTORIAL ESSAY}

noses. Instead NSIP (non-specific interstitial pneumonitis) and AIP (acute interstitial pneumonitis) may be added to a differential diagnosis. ${ }^{2,3,8}$ The end result of these is pulmonary fibrosis that is characterised on HRCT by cysts, honeycombing, parenchymal bands and traction bronchiectasis.

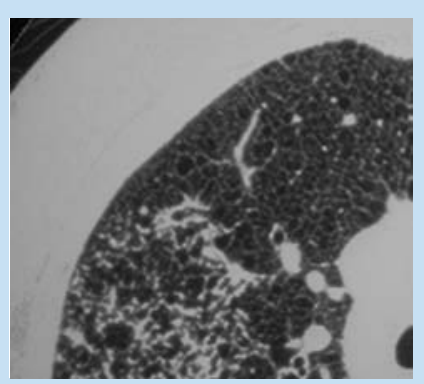

Fig. 26. Fibrosing alveolitis.

\section{Recommended tech- niques for HRCT in children ${ }^{2,3,5}$}

The aim is to produce images of high spatial resolution with no motion artefact using the least amount of radiation possible. Diffuse disease allows for large interslice imaging. Current multi-detector CT allows the acquisition of a volume of contiguous data and allows reconstruction of this data on a high-frequency algorithm in fine slices, thereby yielding both contiguous thicker-slice CT scans and high-resolution CT scans in one event.

mAs: 40 - 80 (Tip: reduces radiation by up to $80 \%$ )

kV: 120 (Tip: an increase from 120 to 140 $\mathrm{kV}$ increases the dose by $40 \%$ )

Slice thickness: $1-2 \mathrm{~mm}$ (multidetector $=$ 1.25) (Tip: thicker slice $=$ less motion artefact)

Slice spacing: $>10$ years $=10 \mathrm{~mm}, 2-10$ years $=7 \mathrm{~mm},<2$ years $=5 \mathrm{~mm}$ (irrelevant for MDCT)

FOV: small as possible, $<25 \mathrm{~cm}$ (Tip: small FOV $=$ higher resolution)

Scan time: shortest possible, e.g. 1- 2 s (Tip: shorter time $=$ less motion)

Reconstruction: High frequency, e.g. bone or lung (Tip: increases edge and detail)

Inspiratory: quiet respiration represents inspiration in children

Expiratory: use decubitus side down (Tip: flat, half or sliver moon trachea $=$ expiration)

Position: only rarely require prone position in children

Viewing: lung window, width $1500 \mathrm{HU}$ and level $-500 \mathrm{HU}$

\section{References}

1. Copley SJ, Coren M, Nicholson AG, Rubens MB Bush A, Hansell DM. Diagnsotic accuracy of thinsection CT and chest radiography of pediatric interstitial lung disease. Am J Roentgenol 2000; 174: 549-554.

2. Koh DM, Hansell DM. Computed tomography of diffuse interstitial lung disease in children. Clin Radiol 2000; 55: 659-667

3. Kuhn JP, Brody AS. High resolution CT of pediatric lung disease. Radiol Clin North Am 2000; 40(1): 89-110.

4. Lucaya J, Le Pointe HD. HRCT features of lung disease. In: Baert AL, Sartor K, eds. Pediatric Chest Imaging. 1st ed. Berlin: Springer-Verlag, 2002: 64 73.

5. Lynch DA, Hay T, Newell JD, Divgi VD, Fan LL. Pediatric diffuse lung disease: Diagnosis and classification using high resolution CT. Am J Roentgenol 1999; 173: 713-718.

6. Evans ED, Kramer SS, Kravitz RM. Pediatric diseases of the lower airways. Semin Roentgenol 1998; 33: 136-150.

7. Kim JS, Muller NL, Park CS. Cylindrical bronchiectasis: diagnostic findings on thin-section CT. Am J Roentgenol 1997; 168: 751-754.

8. Lucaya J, Le Pointe HD. HRCT features in diagnosis of airway disease (4.7). HRCT features in specific lung disease (4.8). In: Baert AL, Sartor K, eds. Pediatric Chest Imaging. 1st ed. Berlin: SpringerVerlag, 2002: 74 - 87.

9. Andronikou S, Wieselthaler N. Modern imaging of tuberculosis in children: thoracic, central nervous system and abdominal tuberculosis. Pediatr Radiol 2004; 34: 861-875.

10. States LJ, Fields JM. Pulmonary haemorrhage in children. Semin Roentgenol 1998; 33: 174-186. 